
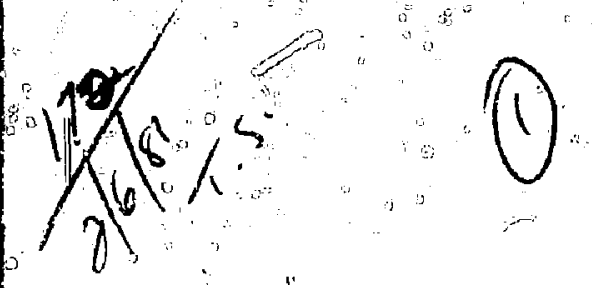

JAMUARY 1981

$P P L-1738$
$v C-209$

$R_{2} 1801$

\title{
LIMITING BETA OF STELLARATORS WITH NO MET CURRENT
}

BY

H. R. STRAUSS AND D, As MONTICELLO

\section{MASTERA}

\section{PLASMA PHYSICS LABORATORY}

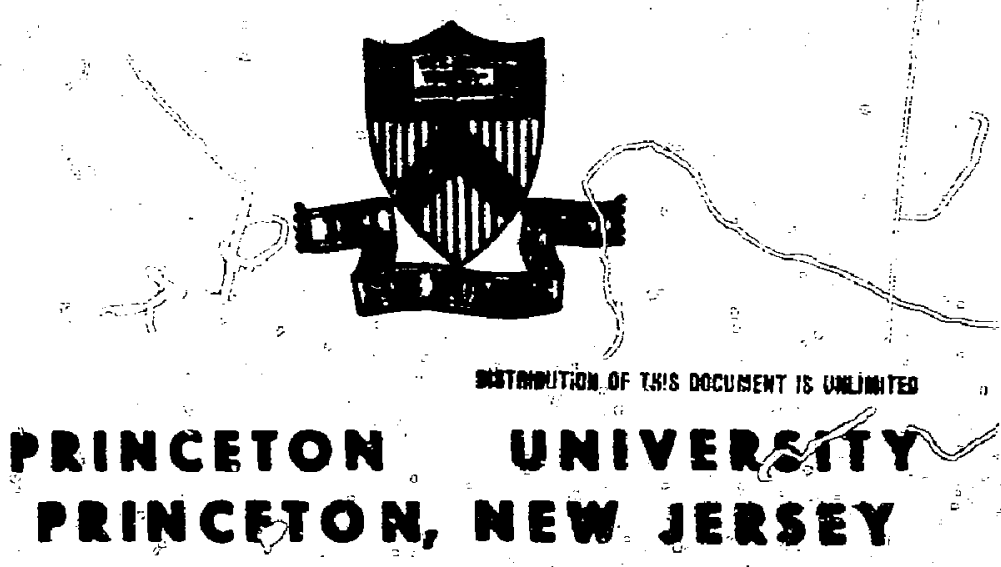

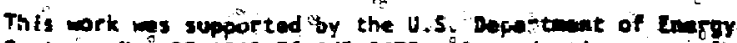
cent raet No. OE-AC02-7S-CNO 3073. Neproduct ten, tranil-

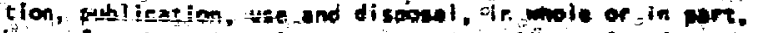
by gr for, the unites states gorerment if merejeted. 
Limititi Beta of Stellarators with no Net Current

H. R. Strauss ${ }^{*}$ and D. A. Monticello

Plasma Fhysics Laboratory, Princeton Unfversity

Princeton, New Jersey 08544

* Courant Inst1tute, for Mathematical Scfences, New York Unfversity

New York, New York 10012

\begin{abstract}
Using reduced nonlinear MAD equations, we find finfte beta, resistive, $\&=2$ stellarator equilibria with no net current. We then investigate stability to low mode number internal MHD modes, and find beta linits comparable to tokamaks. Low shear equilibrla appear to be substantially more stable than $h_{1} 1 \mathrm{gh}$ shear.
\end{abstract}




\section{INTRODUCTION}

An Important advantage of stellarators over tokamaks, is the possibility of steady-state operation. The stellarator rotational transform is then supplied by external windings, rather than by plasma currents which are subject to resistive decay. Even with only a few percent beta, a steady-state device may be economically viable as a fuston reactor. It is nevertheless desirable to attain the highest posstble beta.

These consfderations motivate the present study. We have solved numerically, reduced equations describing nonlinear, resistive, magnetohydrodynamic motion in stellarators. We have obtained flnite beta equilibria, having no net plasma current, consistent with finfte resistivfty. We have concentrated on $\ell=2$ stellarators similar to the low shear Wendelsteln VII experiment, which has operated with no net current, and to the Heliotron $E$, which has much higher shear.

Having obtalned equilibrla, we examine stabllity to ideal MHD (nonreststive) modes, for toroldal mode numbers $n=1,2$, and 3 . We find an Important difference between low and high shear. In both sases, we obtain critical betas comparable to tokamaks, but in the low shear case, there is an accessible upper stable region, suggesting that much higher beta is possible. In high shear $\&=2$ stellarators, there is a strongly unfavorable average curvature which overcomes the stabllizing magnetic well.

\section{I1. REDUCED STELLARATOR EQUATIONS OF MOTION}

The reduced equations are based on the ordering, and methods, of Johnson et al. 1 and Greene and Johnson. ${ }^{2}$ These equations are simflar to those derived for the high beta tokamak ${ }^{3}$ bu contain extra terms for the stellarator 
rotational transform and fleld line curvature. A full derivation of the stellarator equations used in this paper has been given by Strauss. 4

We wrrk with a toroldal coordinate system $(r, \theta, \zeta)$ where $(r, \theta)$ are polar coordinates in the pololdal plane and 5 is the toroidal angle. The major radius is $R=R_{0}+r \cos \theta$, with $r<$ a. We assume the inverse aspect ratto $\varepsilon=a / R \ll 1$. (In practice, $\varepsilon \leq 0.1$.) We assume moderate beta, $\beta \sim \varepsilon$.

The magnetic field, in the lowest order approximation, consists of a constant toroldal field of strength $B_{0}{ }^{*}$ Added to this is the stellarator field of order

$$
\delta=\varepsilon^{1 / 2}
$$

derived from the scalar potential

$$
\phi=B_{0} \sum_{\ell, k}\left(b_{\ell k} / k h\right) I_{\ell}(k h r) \sin \left(\ell \theta+k N_{\zeta}+\ell \theta_{\ell k}\right)
$$

where $I_{\ell}(x)$ is a Bessel function of Inaginary argument: $I_{\ell}(x) \sim x^{\ell}, x<<$;

$b_{\ell k}, \theta_{\ell k}$ are constants, and the stellarator wave 1 ingth 1 s

$$
h=N / R_{0}
$$

In order $E$, the torolaal magnetic fleli contalns corrections from its $\mathbf{R}^{-i}$ varfation and the diamagnetic prissure balance. Also in this order, we find the pololdal field derived from the toroldal plasma curcent which we represent as the curl of the toroldal component of the vector potential $A_{5}$ - Hence, through order $\delta^{2}$, the ragnetic ffeld is 


$$
\begin{aligned}
B=B_{0} \hat{\zeta} & +\delta \nabla \phi-\delta^{2} B_{0}\left(r / R_{0} \cos \theta-p / B_{0}^{2}\right) \hat{\zeta} \\
& +\zeta^{2} \nabla A_{\zeta} \times \hat{\zeta} .
\end{aligned}
$$

However, this is not the most useful representation. The stellarator field contalns a repid $\zeta$ variation, with $N \gg 1$ fundamental periods in the torus. He assume

$$
\mathbb{N} \sim \varepsilon^{-1}
$$

which implies $h \sim a^{-1}$. We are primarily interested in much longer toroidal wavelengths, with mode numbers $n=0,1,2 \ldots$, such that $u \ll N$. Hence we average over the rapldly varying stellarator field. For example, consider solving

$$
B \cdot \nabla_{\psi}=J
$$

We assume $\psi=\psi\left(r, \theta, \zeta_{N}, \zeta_{n}\right)$, where $\zeta_{p j}, \zeta_{n}$ represent the fast and slow toroldal variations, respactively, and

$$
\frac{\partial}{\partial \zeta}=\frac{\partial}{\partial \zeta_{N}}+\delta^{2} \frac{\partial}{\partial \zeta_{n}}
$$

Expanding $\psi=\psi_{0}+\delta \psi_{1}+\ldots$, and using (3), we solve ordex by order. In $0\left(\delta^{\circ}\right)$, we have

$$
\psi_{0}=\psi_{0}\left(\mathbf{r}, \theta, \zeta_{n}\right)
$$


which means $\psi_{0}$ has no rapti $\zeta_{\mathrm{N}}$ varfation. In order $O(\delta)$, we solve

$$
\left(B_{0} / R_{0}\right) \partial \psi_{1} / \partial \zeta+\nabla \psi_{0} \cdot \nabla \phi=0
$$

so that $\psi_{1}$ ts proportional to tha stellarator field. In order $0\left(\delta^{2}\right)$, we average over $\zeta_{\mathrm{N}}$, and we can write the result in terms of the averaged magnetic fleld $\breve{B}$ :

$$
\bar{B} \cdot \nabla \psi_{0}=0
$$

where $\overline{\bar{B}}, \psi_{0}$ depend only on $\left(r, \theta_{,} \zeta_{\mathfrak{a}}\right)$.

Applying this method, we obtain the averaged, reduced, nonlinear equations of motion

$$
\begin{aligned}
\frac{\partial A}{\partial t}=\bar{B} \cdot \nabla u & +n \nabla^{2} A \\
\rho_{0} \frac{d}{d t} \nabla^{2} u & =\bar{B} \cdot \nabla \nabla^{2} A+\nabla \Omega \times \nabla p \cdot \hat{\zeta} \\
\frac{d p}{d t} & =0
\end{aligned}
$$

where

$$
\begin{aligned}
\bar{B} \cdot \nabla & =\partial / \partial \zeta+\nabla \Psi \times \hat{\zeta} \cdot \nabla \\
\frac{d}{d t} & =\frac{\partial}{\partial t}+\nabla u \times \hat{\zeta} \cdot \nabla \\
\psi & =A+\psi_{v}
\end{aligned}
$$




$$
\begin{aligned}
& \Omega=2(r / a) \cos \theta+\Omega_{v} \\
& \psi_{v}=(1 / 2 h \varepsilon) \sum_{\ell, m, k} b_{\ell k} b_{m k} F_{\ell m}(k h r) \cos [(l-m) \theta] \\
& \Omega_{v}=(1 / 2 \varepsilon) \sum_{\ell, m, k} b_{\ell k} b_{m k} G_{\ell m}(k h r) \cos [(\ell-m) \theta]
\end{aligned}
$$

and where

$$
\begin{aligned}
& F_{\ell m}(x)=(m / x) I_{l}^{\prime}(x) I_{m}(x) \\
& G_{\ell m}(x)=I_{l}^{\prime}(x) I_{m}^{\prime}(x)+\left(i m / x^{2}+1\right) I_{l}(x) I_{m}(x) .
\end{aligned}
$$

We have introduced dimensionless variables, expressing $i$ in units of minor radius $a, t$ in units of poloidal Alfvén time $\rho_{0}^{1 / 2} R / B, A$ and $\psi$ in units of $\varepsilon_{0}{ }_{0}$, and pressure in units of $\varepsilon B_{0}^{2}$. In these units, $\beta=2 \varepsilon p$. The density $\rho$, taken constant, is normalized to unity, and $a=1$.

The variables $\psi, A, u, p$ appearing in these equations are averaged quantities, which are independent of $r_{N}$. The stellarator field gives rise to the "vacuum" flux $\psi_{V}$, and the field line curvature $\Omega_{V}$.

If there is no stellarator field, $b_{\ell k}=0$, the equations reduce to those describing a high beta tokamak. 4

The stellarator field contributes to the rotational transform, $1=1 / q$, through $\psi_{v}:$

$$
q \quad=1 / \imath=\int \mathrm{d} \ell /|\nabla \psi|
$$


where the integral is evaluated on a constant surface.

The curvature term $\Omega_{V}$ is generally unfavorable for stability. It persfsts even in the absence of toroidal curvature and can drive interchange modes. It has been shown ${ }^{5}$ that $\Omega_{V}$ is related to the quantity V".

In this paper, we wil assume the plasma is in contact with a rigid conducting wall. We choose boundary conditions $\psi=u=0$. The wall is clrcular in zero order. The shape of the wall in $0(\delta)$ can be found from (1), (4) and is given by

$$
\begin{aligned}
& r / a=1+\sum_{\ell, k} \Delta_{\ell k} \sin (\ell \theta+k N \zeta) \\
& \Delta_{\ell k}=\left(b_{\ell k} / h a\right) I_{\ell}^{\prime}(k h a) .
\end{aligned}
$$

In the following, we will limit consideration to stellarator fields having only one harmonic $\ell, k$. We henceforth set $k=I$ and $d r o p l$ as a subscript.

Prescriblng the boundary shape determines $\ell, N, h$, and $b_{\ell}$, which determines the stellarator field. We prefer to specify $2, N, h$, and ${ }^{2} a$, where ${ }^{a}$ is the vacuum rotational transform at $r=a=1$. In terms of ${ }^{\prime} a$, $v_{v}$, and $\Omega_{v}$ are given by

$$
\begin{aligned}
& \psi_{v}=\left(l_{a} / F_{\ell a}^{\prime}\right) F_{\ell \ell}(h r) \\
& \Omega_{v}=2 h_{a}\left(l_{a} / F_{\ell a}^{\prime}\right) G_{\ell \ell}(h r)
\end{aligned}
$$

where 
$F_{\ell a}^{\prime}=\partial F_{\ell \ell}(h r) / \partial r, r=1$.

For small $h$, we have

$$
\begin{aligned}
& \psi_{v}=\frac{1}{2}\left[\mathrm{r}_{\mathrm{a}} /(\ell-1)\right] \mathrm{r}^{2(\ell-1)}+O\left(\mathrm{~h}^{2}\right) \\
& \Omega_{\mathrm{v}}=2 \mathrm{~h} \psi_{\mathrm{v}}+O\left(\mathrm{~h}^{2}\right) \\
& \Delta_{\ell}=\left[\mathrm{E} \mathrm{a}_{\mathrm{a}} / \mathrm{h}(\ell-1)\right]^{1 / 2} .
\end{aligned}
$$

For silall $h$, an $\ell=1$ field gives $1=0 ; \ell=2$ gives $1=$ const.; and $\ell=3,1 \sim r^{2}$. For $h \geq 1$ an $\ell=2$ field produces a rotational transform with substant:al. shear.

From (24), we observe that given $\varepsilon$ and $\mathrm{l}_{\mathrm{a}}$, we cannot make h too small or $\Delta_{\ell}$ will become too large for our ordering.

III. RESISTIVE EQUILIBRIUM

We are primarily interested in stellarator equilibria consistent with the resistive decay of the plasma current. 6 We seek solutions of Eqs. (5) through (15) with $\partial / \partial \zeta=0$. Instead of $(7)$, we require $p=p(\psi)$ and $\eta=n(\psi)$. This means we are injecting plessure, appropriately, as the plasma evolves. Thus, we solve the two dimensional equations

$$
\begin{aligned}
& \frac{\partial A}{\partial t}=\nabla u \times \nabla \psi \hat{\psi} \cdot \bar{\zeta}+\eta \nabla^{2} A \\
& \frac{d \nabla^{2} u}{d t}=\mu \nabla^{4} u+\nabla\left(\nabla^{2} A\right) x \nabla \psi \cdot \hat{\zeta}+\nabla \Omega \times \nabla p \cdot \hat{\zeta}
\end{aligned}
$$




$$
P=P_{0}\left(\psi / \psi_{0}\right)^{K}
$$

where $\psi_{0}$ is the value on axts. Usually, we choose $\eta=$ const. Viscosity $\mu$ has been added to (26) to dissipate kinetic energy so that an equ111brium can be reached. In equilibrium,

$$
\nabla^{2} A=-(1 / n) \nabla u x \nabla \psi \cdot \hat{\zeta}
$$

Integrating around a flux surface $\psi=$ const, we have

$$
\left\langle\nabla^{2} A\right\rangle=0
$$

where the flux surface average of a quantity $f$ is

$$
\left.\langle f\rangle=\left[\int d \ell f /|\nabla \psi|\right\rceil / \int d \ell /|\nabla \psi|\right\}
$$

and where $d \ell$ is the arc length. This is the well-known result that a stellarator in resistive equilibrium has no net current on each flux surface. 6 It follows from (29) that the total current also vanishes. However, locally there must be some current in order to have momentun balance. If the current is given, (28) requires that $u$ is of order $\eta$. In equilibrium, $u$ may be neglected in (26) for sufficiently small $n$, and we may integrate to obtain

$$
\nabla^{2} A \neq-\Omega \frac{d p}{d \psi}+g(\psi)
$$


This is the equilibrium equation of Greene and Johnson. ${ }^{2}$ However, $g(\psi)$ is determined by (29) which gives

$$
\nabla^{2} A=\lceil\langle\Omega\rangle-\Omega] \frac{d p}{d \psi}
$$

This is a generalized partial differential equation, as discussed by Grad et al. ${ }^{7}$ Methods have been found for solving equations of this type, iterating between solutions of Polsson's equation on the left hand side and the calculation of the flux surface averages on the right hand side. Although we do not employ such methods here, it is important to note that the solutions of such equitions, in practice, are unique. Hence specifying $\psi_{v}$ and $p(\psi)$ serves to determine a generally unique equilibrium, regardless of initial conditions, the form of $\eta(\psi)$, and the magnitude of $\eta$ and $\mu$, providing $\eta$ is sufficiently small. This has been verified In our numerical work.

It is instructive to examine the energy conservation law associated with Eqs. (25) - (27). Assuming $\psi=u=$ const. at $r=1$, and defining the energy

$$
E=\frac{1}{2} \int d^{3} x\left\lceil(\nabla \mathrm{A})^{2}+(\nabla \mathrm{u})^{2}-2 \Omega \mathrm{p}\right\rceil
$$

we obtain

$$
\frac{\partial E}{\partial t}=-\int \lambda^{3} x\left[n \nabla^{2} A\left(\nabla^{2} A+\Omega \frac{d p}{d \psi}\right)+\mu\left(\nabla^{2} u\right)^{2}\right]
$$

The term $\Omega d p / d \psi$ would not appear if there were no pressure sources, i.e., if $\delta p / d t=0$. Neglecting $n^{2} u$, we verify that $\partial E / \partial t=0$ in equilibrium.

We now present approximate analytic solutions of Eq. (32). Assuming small $\beta$ and $h$, we find expressions for the rotat:onal transform and for the 
shift of the magnetic axfs, assuming a single hacnonfc stellarator fleld. Unlike Johnson et. al, we assume $\psi=$ const. on the boundary $r=1$. Hence, we do not find a class of solutions with negative shift of the magnetic axis. Also with our boundary conditions, we would need to introdice more stellarator harmontes \& In order to obtain Scyllac type ${ }^{B}$ solutions.

From (14), (20), we obtaln for small h,

$$
\psi_{v}=\frac{{ }^{1} a}{2(l-1)+h^{2}}\left[\mathbf{r}^{2(l-1)}\left(1+\frac{h^{2} r^{2}}{2 h}\right)-\left(1+\frac{h^{2}}{2 \ell}\right)\right] .
$$

We find

$$
l / l_{a}=\left[\frac{2(l-1)+h^{2} r^{2}}{2(l-1)+h^{2}}\right] r^{2(l-2)} .
$$

We now solve (32) by expanding in $\beta$ :

$$
\psi=\psi_{0}+\beta \psi_{1}+\cdots,
$$

In zero order, $\psi_{0}=\psi_{\mathrm{v}}(r)$. In first order,

$$
\Omega_{v} P^{\prime}\left(\psi_{v}\right)+g_{1}\left(\psi_{v}\right)=0
$$

$$
\nabla^{2} A_{1}+2 r \cos \theta p^{\prime}\left(\psi_{\nabla}\right)=0
$$

Consider a pressure piofile of the form (27).

If $K=1, p^{\prime}=-p_{0} / \psi_{v}(0)$. Then 


$$
A_{1}=\frac{P_{0}}{4 \psi_{v}(0)}\left(r^{3}-r\right) \cos \theta .
$$

To find the magnetic axis, we solve

$$
\nabla\left(\psi_{v}+A_{1}\right)=0
$$

Taking the terms with the lowest powers of $r$ in $\psi_{v}$ and $A$, we find that the magnetic axis is located at $r=r_{m}, \theta=0$, where

$$
r_{m}^{2 \ell-3}=\frac{P_{0} q_{a}^{2}}{2} \frac{\left[i+h^{2} / 2(l-I)\right]^{2}}{1+h^{2} / 2 g}
$$

and where $q_{a}=1 / 1_{a}$.

When $\ell=2$, the displacement of the magnetic axis is proportional to the poloidal beta $\beta_{p}=B q_{a}^{2} / \varepsilon^{2}$. When $\ell=3, r_{m}$ is larger and scales with $\left(B_{p} \varepsilon\right)^{1 / 3}$.

IV. NUMERICAL RESULTS

We solve Eqs. (5) through (15) and (26) through (30) numertcally using STELLA, a modification of the Frinceton high beta tokamak code HIB. 9,10 The equations are Fourfer analyzed in the angles $\theta$ and $\zeta$, and tinite dffferenced In $r$. The resulting couplad mode equations are time advi. $c \_d$ by a two level predictor corrector method. Convolution sums are performed directly, taking advantage of the vector processing it the MFE CRAY-I computer. Typically we used 50 radial mesh points, with 6 poloidal harmonics for equilibrium calculations, and 15 polotdal harmonics for stabllity. 
To find equilibria, we start with an Initial cylindrical state with $\psi=\psi_{v}(r)$ and the pressure given by (27). The equatinns are time advanced unt11 a steady state is reached.

Fig. I shows the constant pressure (and flux) surfaces for an equilibrium with 1nput parameters $\ell=2$, ha $=0.25, q_{a}=1.80$, and $P_{0}=0.13$. These parameters correspond to the Wendelstein VII experiment. 11 Recall that in our ¿̇mensionless units

$$
B=2: p \text {. }
$$

The pressure is quadratic in $\psi, K=2$. The average pressure is about $0.4 \mathrm{P}_{\mathrm{o}}$. Note the typical finlte beta outward shift of the magnetic axio. is mentioned above, more stellaracor harmonics would be required for an inward shitit in this case, an add $t$ tonal $\ell=1$ or $\ell=3 \mathrm{fteld}$.

Contours of constant toroidal current density are shown 1n Fig. 2. The separatrix which divides the regions of posftive and negative current passes through the magnetic axis as requi,red by Eq. (29). The total current vanishes to an accuracy of about $10^{-3}$.

The current profile along the major radius is given in Fig. 3. As in the high beta toldmak, the current exhibits peaking near the outer edge of the plasma. However, the magnitude of the current is substantially less than for a comparable tokamak.

The rotational transform $1=1 / q$ is one of the main factors determining stability. The q profile is shown in Fig. 4. Due to the small value of ha, the profile is almost flat. It is noteworthy that the peak value of $q$ occurs at nefther the magnetic axis or the wall. These features of the q profile w11l prove important for interpreting our stability results for equilibria of this type. Note that the safety factor at the wall is not exactly equal to $q_{a}$, since $q_{a}$ refers only to the vacuum field. 
For small beta and ha, the shift of the magnetic axis agrees well with Eq. (28), for the linear pressure profile, $K=1$. Figure 5 compares the results for parameters $\ell=2$, ha $=0.25$, and qa $=1.8$ with $r_{m}$, the radial displacement of the magnetic axis, plotted as a Eunction of $P_{0}$.

In Fig. 6, we show the $q$ profile of another equilibrium, with input parameters $\ell=2$, ha $=1.8$, qa $=0.555, P_{0}=0.2$, and $K=2$. Dise to the rather large value of ha, there is substantial. shear, and finite beta effects are less important. The contours of $\mathrm{p}, \psi$, and current are qualitatively simflar to the low shear $\ell=2$ case. These parameters correspond to the Hellotron- $E^{12}$ experiment, which has $\varepsilon=0.1$. The magnetic axis shtfe as a function of $P_{0}$ is shown in Fig. 7 .

We now consider the stability of finite beta, $l=2$ stellarator equilfbria with no net current, haviı? parameters consistent with those considered above. To find unstable efgenmodes, the averaged equations of motion (5), (6), (7) are linearized. The equilibrium quantities are independent of $\zeta$, so the efgenmodes have $\zeta$ dependence exp $(\ln \zeta)$. The fastest growing instability foi a given equilibrium and toroidal mode number $n$ can be found by integrating the equations in time. We consfder only ideal, non resistive modes, with $\eta=0$ in Eq. (5). We also neglect the equilibrium resistive flow.

We first examine the low shear case, ha $=0.25$. Figure 8 shows contours of the perturbed velocity potentlal $u$, for an $n=3$ instabjlity of an equilibrium with $q_{a}=1.8, P_{0}=0.13$. Referring to Fig. 4, we note that the mode is concentrated near the flux surface of maximum $q$, which is close to 2. The dominant polofial mode number is $m=6 \approx n q$. It is interesting that there is instability without an exact rational surface in the plasma, since $q$ $<2$. 
Figure 9 shows the squared growth rate in units of the pololdal Alvfen time: as a function of $P_{0}$, with parameters $q=2$, ha $=0.25$, qa $=$ 1.85. These equilibria have $K=2$, which means the pressure ts quadratic In $\psi$. Curves are plotted for toroidal mode numbers $n=2,3$. Just as in the high beta tokamak, there is a second stability region due to the magnetic ve11. The lower critical $B$ is $0.2 \mathrm{E}$, which is comparable to a tokamak, ${ }^{9}$ but the upper critical $B$ is only $0.4 \mathrm{E}$. The growth rate is of order $0.1 \mathrm{~B} / \mathrm{\rho}_{\mathrm{O}}^{1 / 2 \mathrm{R}}$ rhich is about the same as was found in tokamaks. Hence equilibria of this type have good stability.

At low shear, stability depends critfcally on the rational surfaces, at which $q=m / n$. For low $n$ there is only one rational surface in or near the plasma. Recall that in these equilibria, q varles by only a few percent, with the maximum $q$ somewhat larger than $q_{a}$. Fig. I0 shows the effect of varying $q_{a}$, with $\ell=2$, ha $=0.25$, and $p_{0}=0.13$, whlch corresponds to the middle of the unstable range of Fig. 9. The curves show the growth rate squared for $n=$ $1,2,3$ modes, as a function of $q_{a}$. For $n=3$, there are rational surfaces at $q$ $=1.667,2.0,2.333,2.667$, with $m=5,6,7,8$. Above the $n=3$ curve are shown the dominant pololdal mode number $(m)$ of the most unstable mode. The maximum growth rates are correlated with the minfmum values of $|\mathrm{m}-\mathrm{nq}|$. The results are stmilax for $\mathrm{a}=1$ and $\mathrm{n}=2$.

If we consider modes with $n$ less than or equal to 3 , we see that there are especially favorable $q$ values for stability. In Fig. 11 we plot the growth rate squared as a function of $\beta$, for $q_{a}=1.70$. With this choice of $\mathrm{q}_{\mathrm{a}}$, the $\mathrm{n}=1,2,3$ modes are completely stable. The mode frequency reaches its minimum at $B=0.4 \varepsilon$, which is higher than the most unstable $B$ at $q_{a}=1.85$. of course, for very large $n$, the rational surfaces become dense, and there should be no gaps in the range of unstable $\beta$ values. It may be 
legltimate to look only at low $n$, however, if finlte Larmor radius effects are 1mportant. In any case, a key assumption of the present theory is that $n$ is much less than $N$, the number of stellarator periods, and typically $N$ is of order 10. Hence large $n$ results would be of dublous validity. In Wendelstein VII, $N=5 ;$ in Heliotron $E, N=18$.

Turning now to the high shear $\ell=2$ equilibrla, we find a rather different stability picture. We consider parameters $\&=2$, ha $=1.80, q_{a}=$ 1.80, with a quadratic pressure proftle, $K=2$. Referring to Fig. 6, we note that there are typically several rational surfaces in the plasma. F1gure 12 shows the squared growth rate as a function of $B$, $f(x) n=1,2,3$. The critical $\beta=0.33 \mathrm{E}$. There is no second stability region. This may be understood by recalling from Fq. (20) that the stellarator curvature $\Omega_{v}$, which is unfavorable, is proportional to ha. Hence it is 7.2 times larger than in the prevfous low shear case. The stabflizing magnetic well, caused by the outward shift of the magnetic axis, is insufficient to balance the stellarator curvature and produce a second st: ble region.

Our results suggest that lo:y shear is desirable for $\ell=2$ finite beta stellarators with no net current, for which $\mathrm{l}$ is chosen to exclude low order rational surfaces from the plasma. However the stellarator wavelength $h$ which governs the shear, may not be made arbitrarily small, if $q_{a}$ and $\varepsilon$ are fixed. As shown by Eq. (24), the outer flux surface distortion can become large, so that our ordering breaks down. Given this constrafnt, it seems worthwhile to keep ha, and thus the shear and $\Omega_{\nabla}$, as small as possible. Nonetheless, the high shear $\ell=2$ stellarator has a critical beta comp:icable to a tokamak. Add to this the potential for steady-state operation, and the stellarator seems, once agatn, a reasonable candidate for a future fuston reactor. 
ACKNOWLEDGENT

The authors thank J. L. Johnson, T. K. Chu, P. Garabediari, and o. Betancourt for helpful discussions. One of us (H.S.) thanks P. H. Rutherford and R. C. Grimm for making possible a recent visit to Princeton Plasma Physfes Laboratory, where this work was performed.

This research was supported by the United States Department of Energy under contract no. DE-ACO2-76-CHO3O73. 


\section{REFERENCES}

1. J. L. Johnson, C. R. Oberman, E. M. Kulsrud, and E. A. Frieman, Phys. F1uids $1,281,(1958)$.

2. J. M. Greene and J. L. Johnson, Phys. Flu1ds 4, 875, (1961).

3. H. R. Strauss, Phys. Fluids 20, 1354, (1977).

4. H. R. Strauss, Plasma Physics 22, 733, (1980).

5. J. H. Greene, J. L. Johnson, and K. A. Weimer, Plasma Physics $\underline{8}, 145$, (1966).

6. M. D. Kruskal and R. M. Kulsrud, Phys. Fluids 1, 265, (1958).

7. H. Grad, P. N. Hu, and D. C. Stevens, Proc. Nat 1. Acad. Sc1. USA $\underline{72}$, 3789 , (1975).

8. J. P. Freidberg, R. Y. Dagazian, and D. C. Barnes, Phys. Fluids 22, 926, (1979).

9. H. Strauss, พ. Park, D. A. Monticello, R. B. White, S. C. Jardin, M. S. Chance, A. M. M. Todd, and A. H. Glasser, Nuc1. Fusion 20, 628, (1980).

10. D. A. Monticello, W. Park, S. C. Jardin, M. S. Chance, R. L. Dewar, R. B. White, R. C. Grimm, J. Manickam, H. R. Strauss, J. L. Johnson, J. M. Greene, A. H. Glasser, P. K. Kaw, P. H. Rutherford, and E. J. Valeo, 8th International Conference on Plasma Physics and Controlled Nuclear Fusion Research, I, A.E.A., Brussels (1980).

11. W VII Team, 8th International Conference on Plasma Physics and Controlled Nuclear Fustion Research, I.A.E.A., Brussels (1980).

12. K. Uo, A. Ifyosh1, T. Obik1, S. Morimoto, M. Wakatani, A. Sasak1, K. Kondo, 0. Motojima, M. Sato, K. Hanatanf, T. Mutoh, H. Zush1, H. Kaneko, I. Ohtake, M. Nakasuga, T. Mizuuchi, S. Kinoshita, and 
Y. Nakashimo, 8th International Conference on Plasma Physics and Controlled Nuclear Fuston Research, I.A.E.A., Mrussels (1980). 
FIGURE CAPTIONS

Fig. 1. Contours of constant $p(\psi)$ for a zero net current stellarator equilibrium with $\ell=2$, ha $=0.25, q_{a}=1.7$, and $\beta=0.26 \varepsilon$. These parameters correspond to the Wendelsteln VII experiment, which has $\varepsilon=0.05$.

Fig. 2. Contours of constant toroldal current density of the equilibrium of Fig. I. The separatrix, dividing posttive and negative current reglons, passes through the magnetic axis. The net current on each Elux surface vanishes due to $\mathrm{E}_{\mathrm{q}}$. (28).

F1g. 3. Trroldal current profile as a function of $r$, along the major radius. The current is peaked more in the outer (large $R$ ) part of the plasma.

Fig. 4. Rotational transform profile as a function of $r$ along the major radius, for the equilibrium of F1g. 1. Due to the small value of ha, $q(\psi)$ is almost flat. The peak $q$ occurs at netther the magnetic axis nor the plasma boundary.

Fig. 5. Displacement of the magnetic axis as a function of $B$, for magnetic parameters $\ell=2$, ha $=0.25, \mathrm{q}_{\mathrm{a}}=1.8$. The displacement tends to saturate at large beta. At low beta, there is good agreement with Eq. (37), also shown, which gives a displacement linear in beta. 
Fig. 6. The $q$ profile of a different equilibrium, with $\ell=2$, ha $=1.8$, $q_{a}=0.555$, and $B=0.4 \varepsilon$. The magnetic field parameters are similar to the Helfotron $E$ experiment, which has $E=0.1$. With large ha, there is substantial shear. The peak $q$ occurs at or near the magnetic axis.

F1g. 7. Magnetic axis shift $r_{m}$ as a function of $P_{0}=\beta / 2 \mathrm{E}$ for ha $=1.8$ equilibria. The solid line is the analytic result for small $P_{0}$.

F1g. 8. Contours of perturbed velocity potertial $u$, of an $n=3$ Iinear efgenmode of an equilbrium with ha $=0.25, q_{a}=1.8, p_{0}=0.13 \varepsilon_{\text {. }}$

Fig. 9. Linear stability of equilibria with $\ell=2$, ha $=0.25, q_{a}=1.85$, as a isnction of $\beta / \varepsilon$, and for toroldal mode numbers $n=2,3$. There is a high beta stablitty region due to the magnetic well. The critical (peak) betas are $0.2 \varepsilon$ and $0.4 \varepsilon$.

Fig. 10. The effect of varying $q_{a}$. The squared growth rate is shown for $n=1$ (dotted line), $\mathrm{n}=2$ (dashed line), $\mathrm{n}=3$ (solid line). Beta is $0.26 E$ in the mfddle of the unstatle range of Fig. 8 . The dominant poloidal mode number of the most unstable mode is also shown. Instability is correlated with the presence of a rational surface $q=m / n$ in the plasma.

Fig. 11. Growth rate squared as a function of beta, for $n=1,2,3$ modes. The equilibria are almost the same as In Fig. 8, except $q_{a}=1.7$. This Is sufficlent to stabilize the modes. 
F1g. 12. Stablity of equilibrla wtth $l=2$, ha $=1.8, q_{a}=0.555$. The growth rate squared 1 s shown as a runction of beta, for $n=1,2,3$ raodes. The critical beta $1 \mathrm{~s} 0.33 \mathrm{E}$, and there is no high betal stability. Unlike the previous low shear case, there are always rational surfaces in the plasma, and the unfavorable stellarator curvature, $\Omega$, is much larger. 


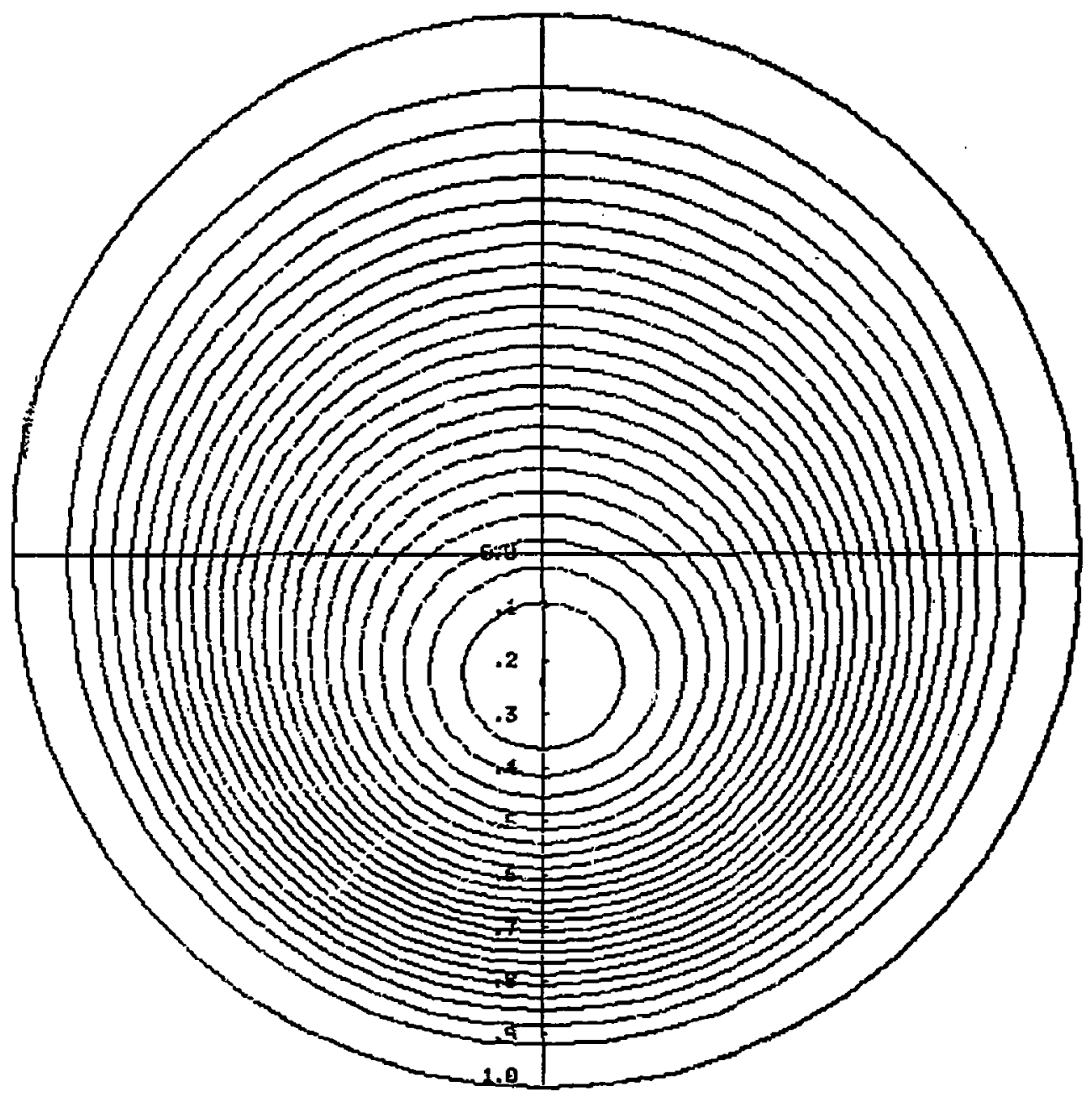

Fig. 1. (PPPL-802320) 


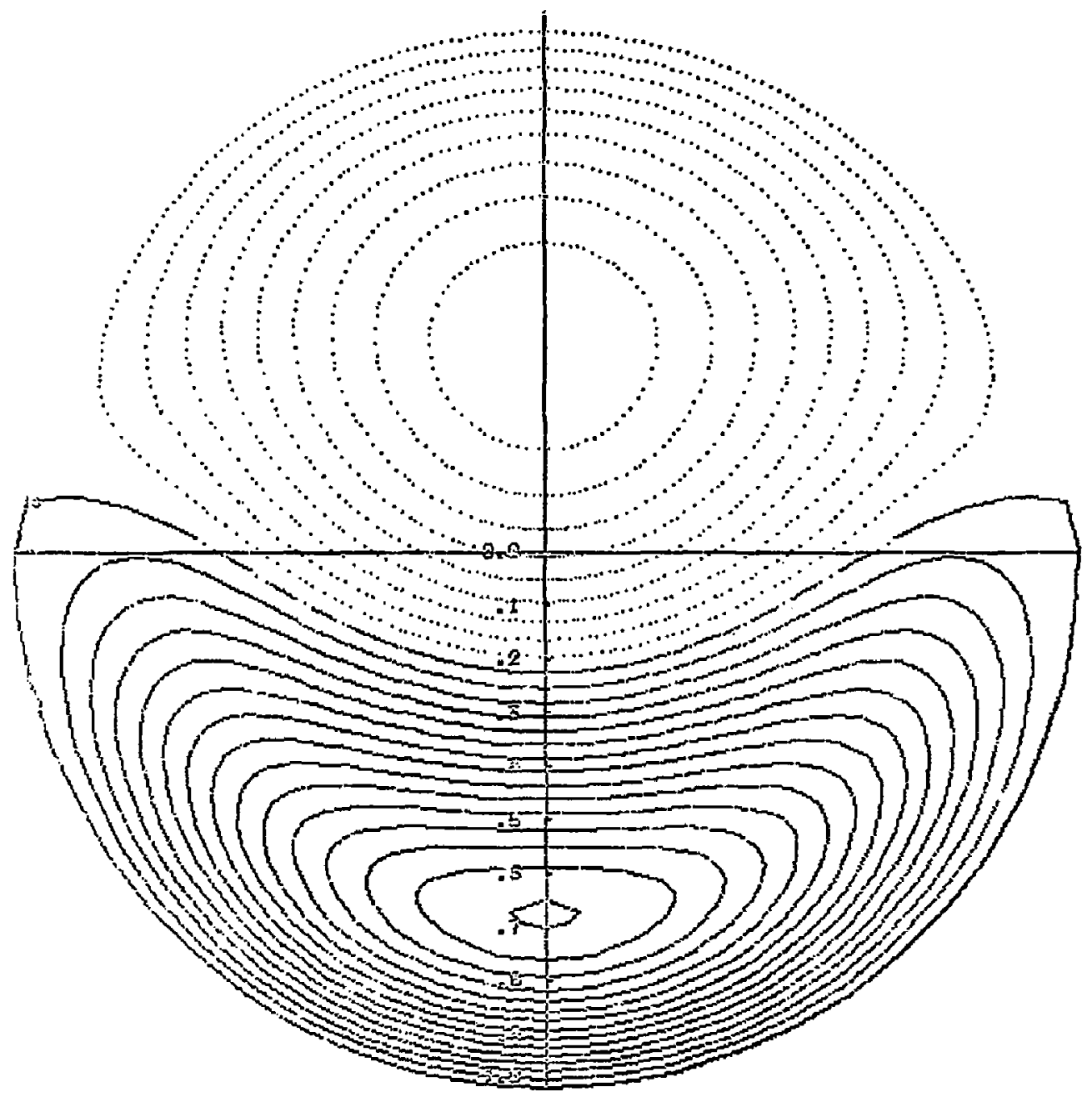

Fig. 2. (PPPL-802318) 


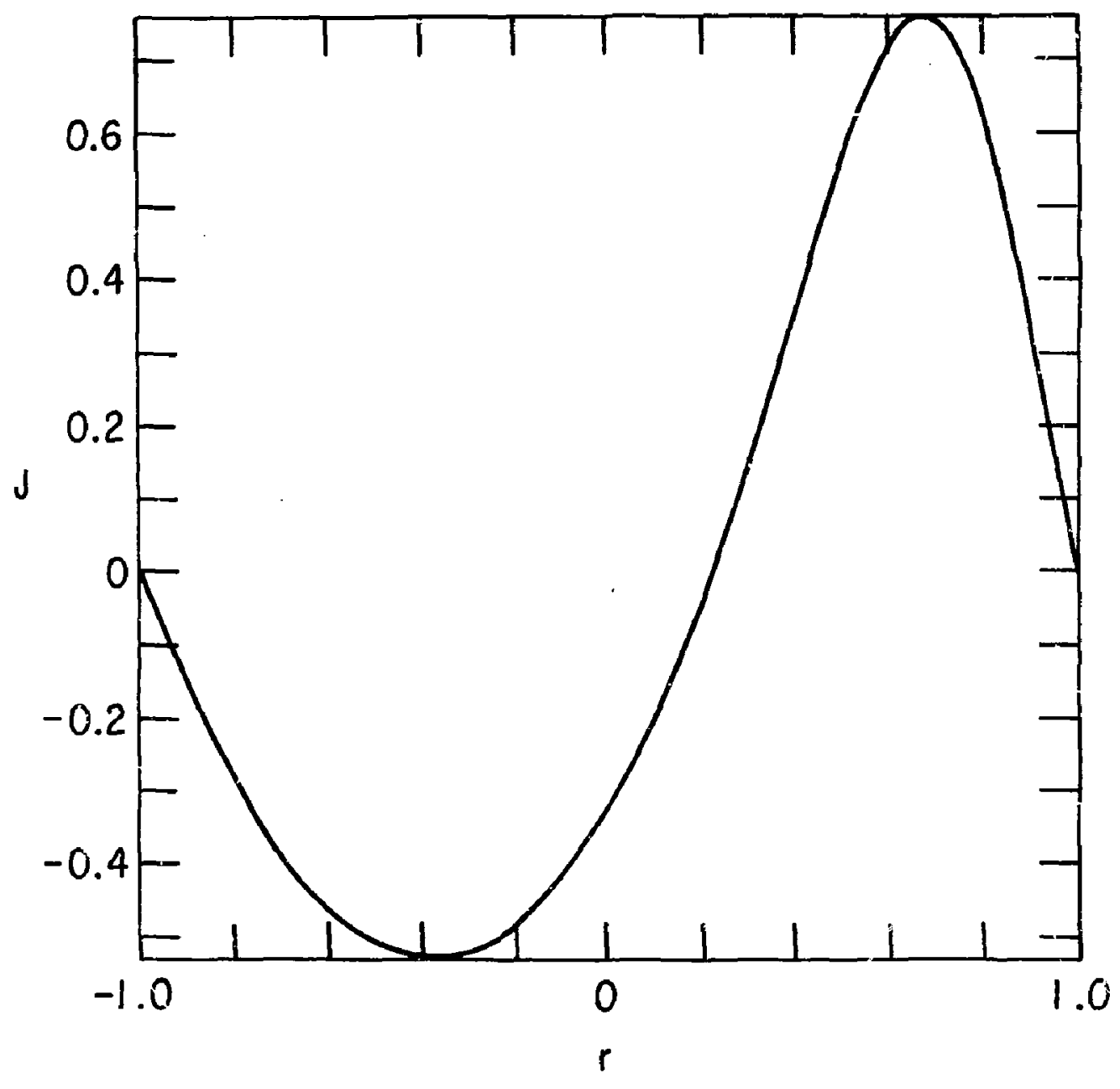

Fíy. 3. (PPEL-8023:3) 


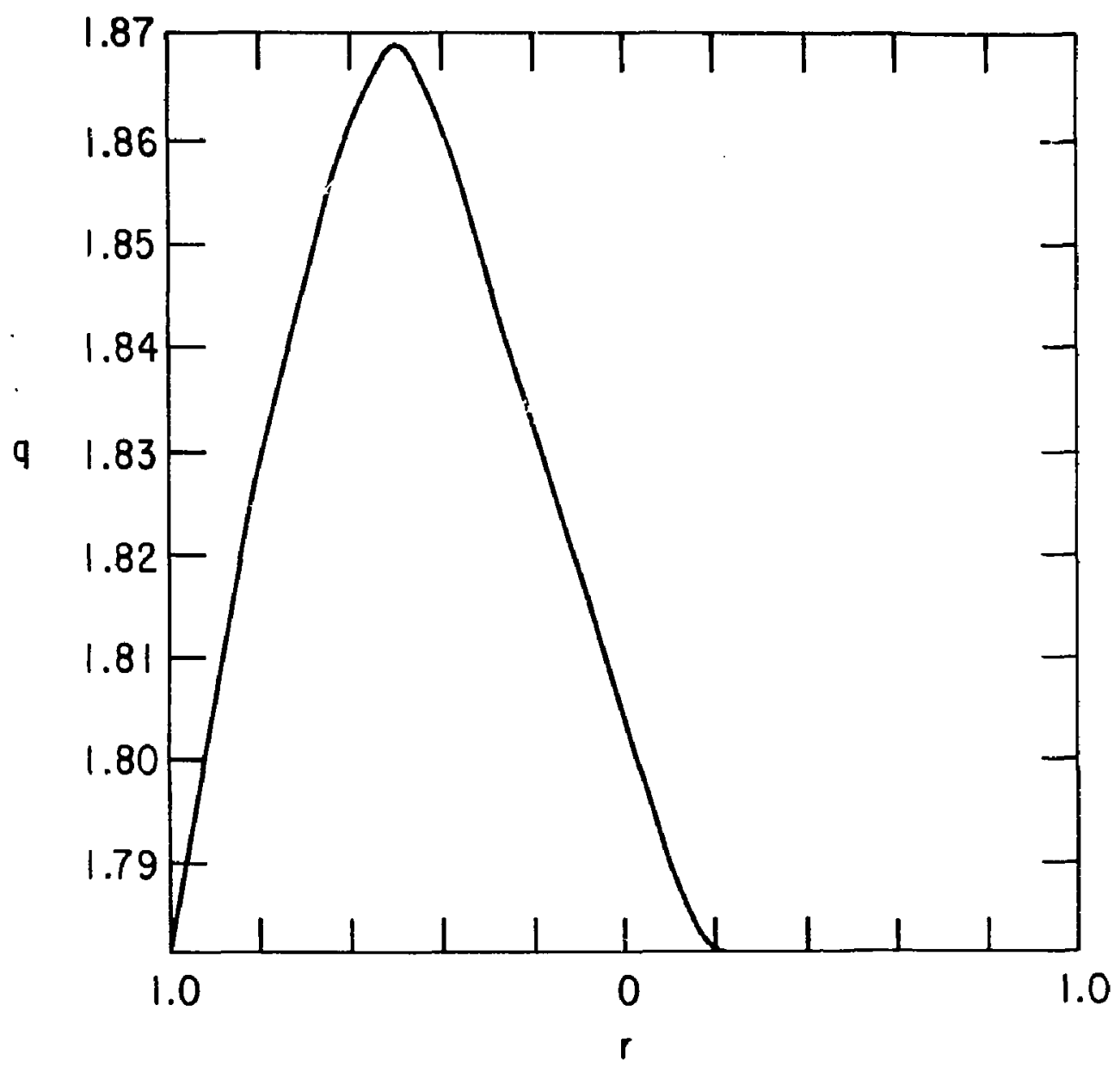

Fig. 4. (PPPL-802412) 


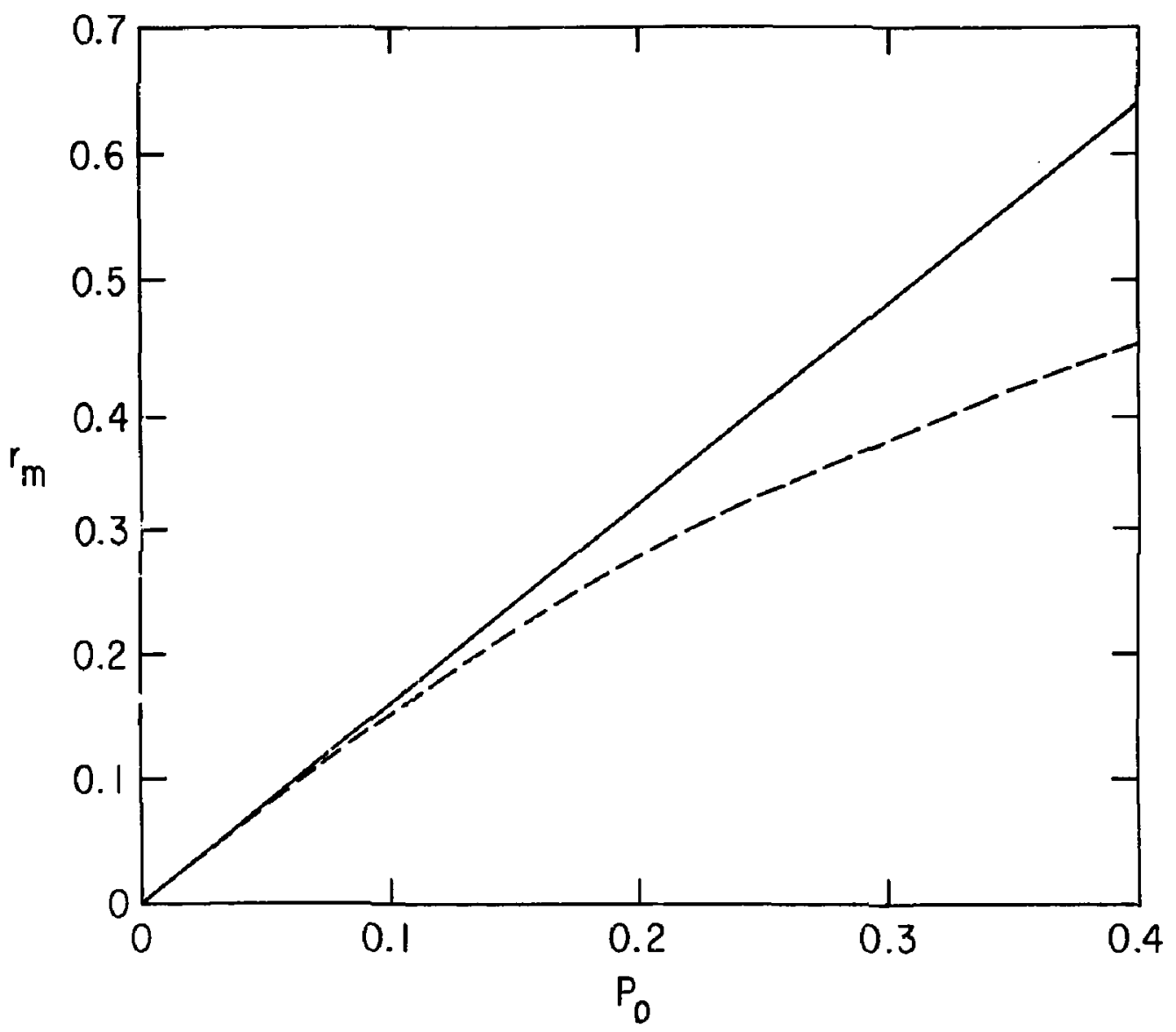

Fig. 5. (PPPL-802331) 
$-28-$

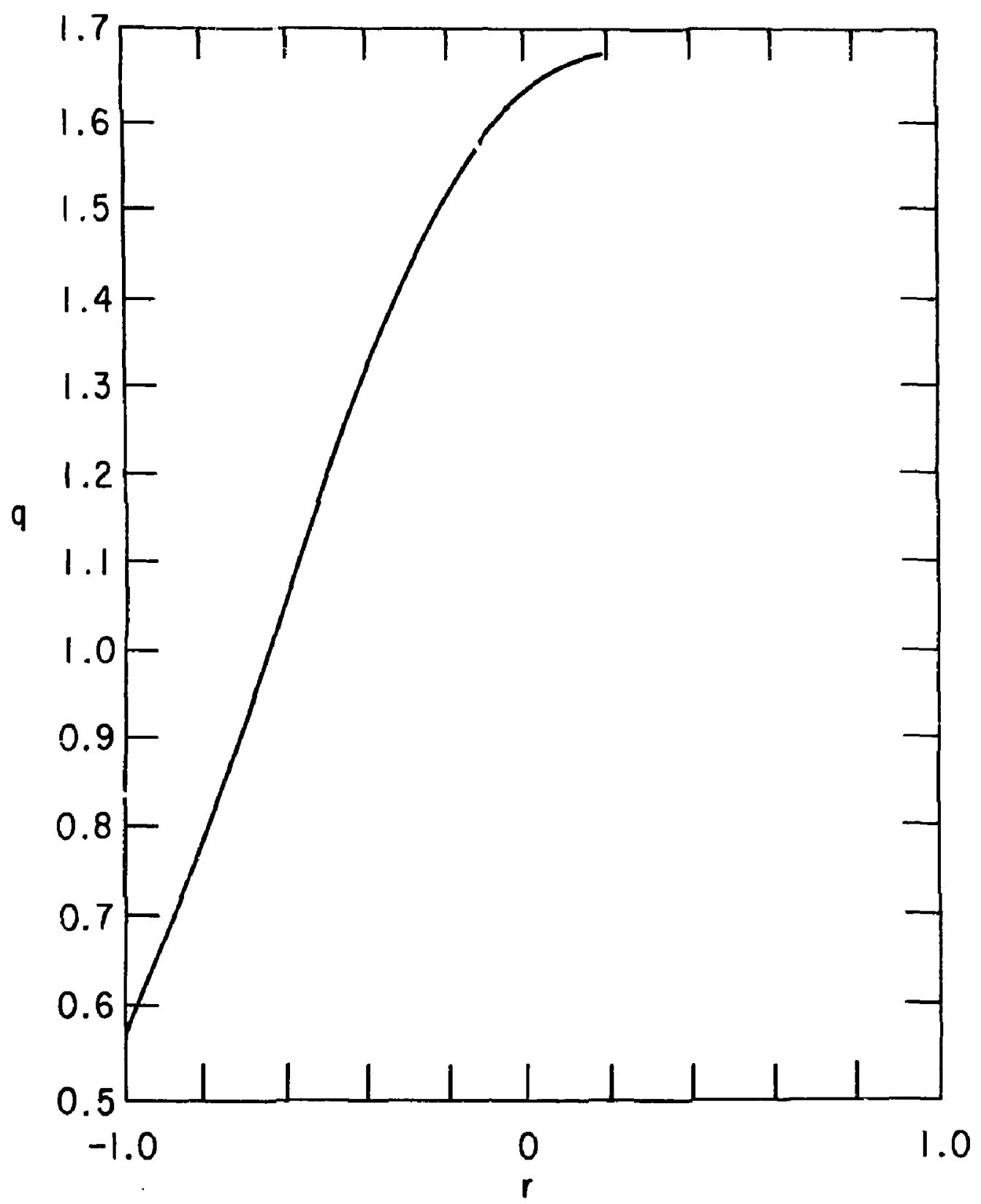

Fig. 6. (PPPL-802332) 


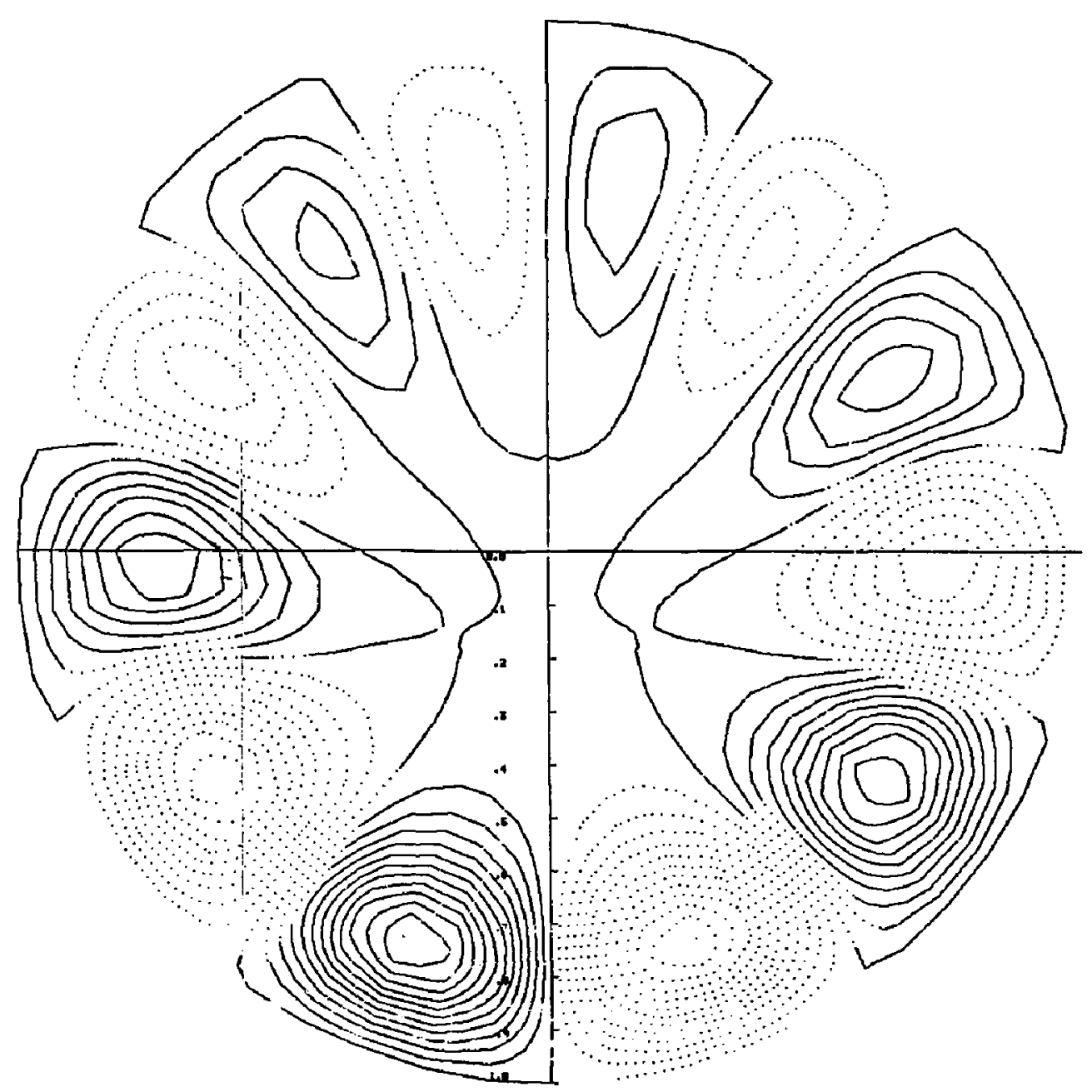

Fig. 8. (PPPL-802321) 


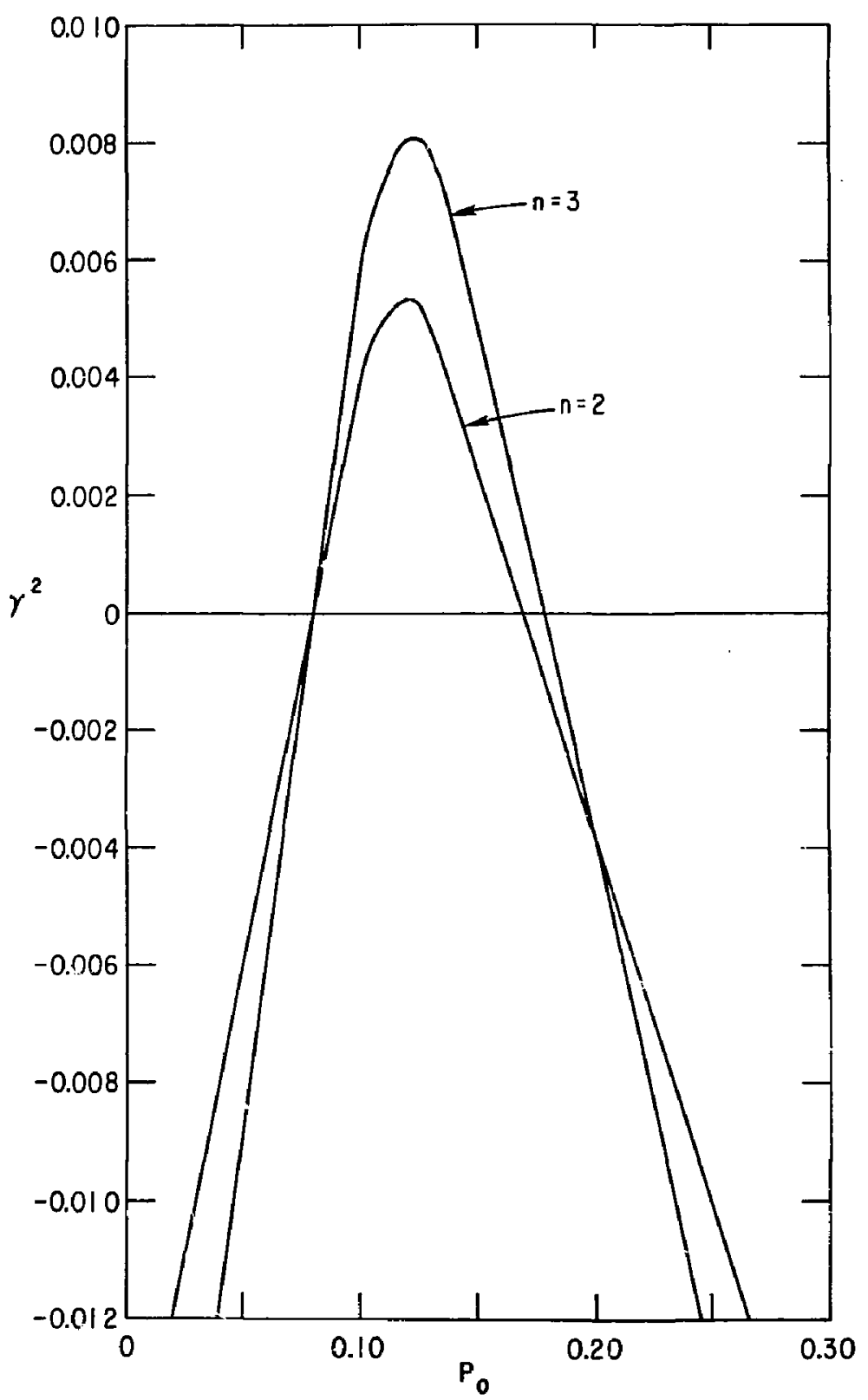

Fig. 9. (PPPL-802413) 


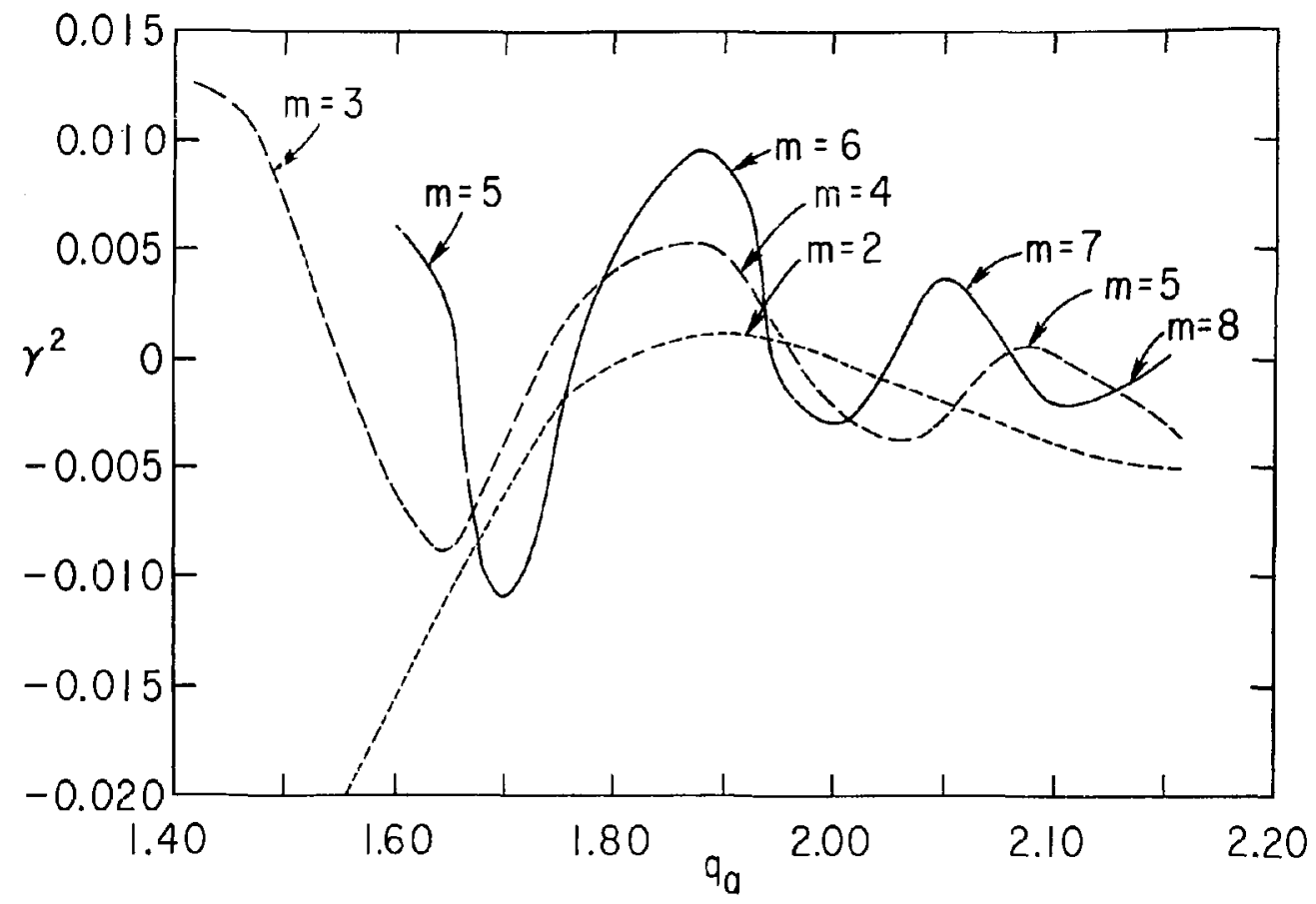

Fig. 10. (PPPL-802410) 


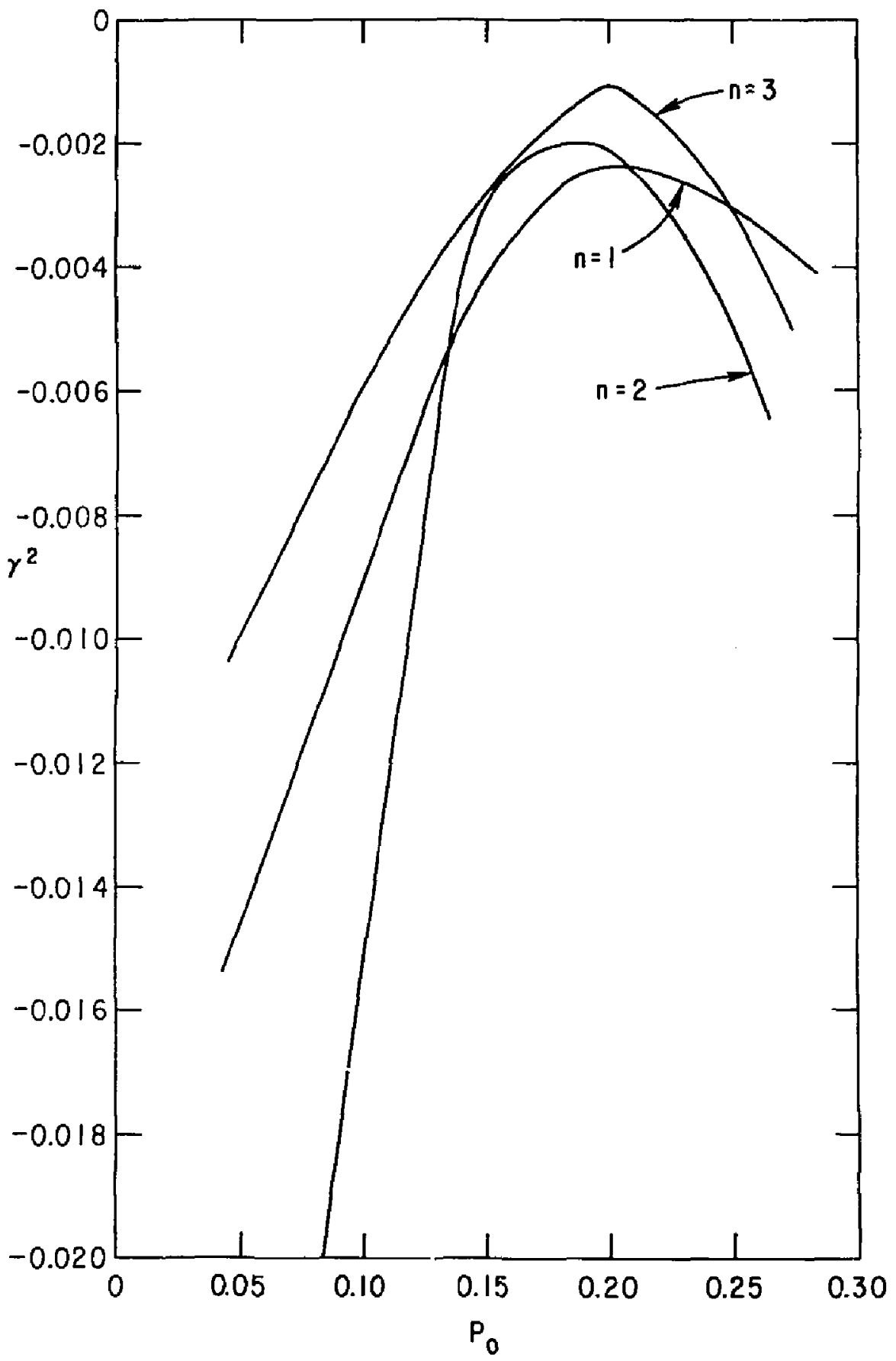

Fig. 11. (DPPL-802414) 


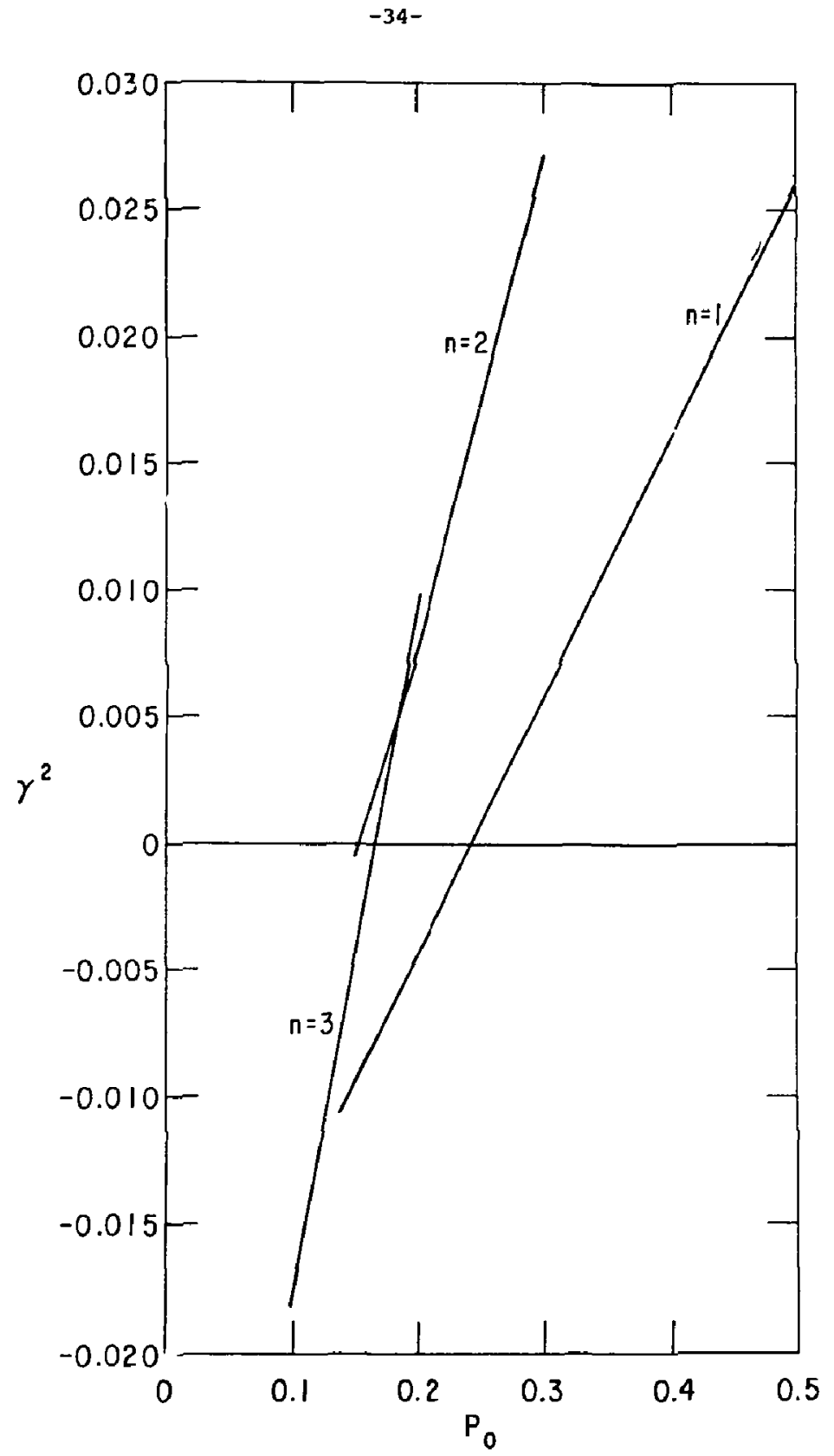

Fig. 12. (PPPL-802411) 